\title{
Review
}

\section{Primary prevention of Type 2 (non-insulin-dependent) diabetes mellitus}

\author{
H. King and J.E. Dowd \\ Division of Noncommunicable Diseases, World Health Organization, Geneva, Switzerland
}

\begin{abstract}
Summary. Type 2 (non-insulin-dependent) diabetes mellitus is the major form of the disease in all societies. Its public health impact appears to be increasing and the greatest genetic predisposition to the disease is encountered in developing communities. The reduction or elimination of disease in whole populations is a fundamental goal in public health. Whilst several factors are associated with the development of Type 2 diabetes, it is not clear how they cause the disease, if indeed they do, nor whether they act in the same way in all populations. Risk factors may be true determinants of a disease but alternatively they may be associated with its occurrence only by virtue of an innocent relationship with the true causes. Furthermore, known risk factors usually explain only a small proportion of any chronic disease. The role of risk factors in disease causation is therefore of fundamental importance in considering disease prevention. Two alternative strategies for prevention of disease in populations have been proposed. The population strategy seeks to remove the
\end{abstract}

causes of disease in communities as a whole, whilst the highrisk strategy aims to identify subjects at increased risk, and to intervene selectively. The population approach should be tried and carefully evaluated in selected communities at above-average risk of several noncommunicable diseases. However, certain epidemiological features of Type 2 diabetes, including the distributional characteristics of glycaemia and the complications of hyperglycaemia, the clustering of cardiovascular risk factors in the diabetic subpopulation, as well as uncertainties over the causal nature of known risk factors, suggest that a high-risk approach to prevention is also appropriate. Optimal allocation of resources to the two approaches requires a detailed knowledge of the disease process in individual communities.

Key words: Type 2 (non-insulin-dependent) diabetes mellitus, preventive medicine, epidemiology, risk.
Primary prevention aims to reduce or even eliminate disease incidence in whole populations. For diseases which are already established in the community, it represents a fundamental objective in public health. In the case of the major noncommunicable diseases such as cardiovascular disease and cancer, the presently recommended strategy includes personal and community efforts aimed at improving nutritional status, enhancing physical fitness and maximizing environmental safety. It is generally believed that integrated, community-based intervention aimed at altering recognised social and behavioural health hazards have the greatest potential for the primary prevention of the major noncommunicable diseases, including diabetes $[1,2]$. In the past few years, the World Health Organization (WHO) has produced reports on the primary prevention of both essential hypertension [3] and coronary heart disease [4]. In the near future, the prevention of diabetes may also be a subject for consideration by a WHO Study Group.
Type 2 (non-insulin-dependent) diabetes is the most common form of the disease in all communities. Not only a burden to industrialized countries, it appears to be having an increasing impact on the public health of developing countries. Careful consideration of the possibility for the primary prevention of Type 2 diabetes in whole populations is therefore timely. An understanding of the epidemiology of the disease is important for the development and implementation of appropriate preventive strategies.

\section{Genetic factors and Type 2 diabetes}

So far, research has failed to identify specific genetic markers which confer susceptibility to Type 2 diabetes in most populations. However, the disease is known to be familial [5]. It is also thought that a high degree of metabolic efficiency has been evolutionarily advantageous to populations that have traditionally lived in harsh and unstable 
environmental circumstances [6]. As a consequence such populations, which include those of the Indian subcontinent, indigenous North Americans and Pacific islanders, appear to be more predisposed genetically to abnormalities of glucose metabolism, once they adopt a western diet and lifestyle. Thus, it is in the developing world that the greatest underlying susceptibility to Type 2 diabetes is encountered. There is evidence that Type 2 diabetes may be determined by a major gene(s) in some of these populations $[7,8]$ though a polygenic mode of inheritance may yet prove to determine glucose tolerance in the majority of communities.

\section{Environmental factors and Type 2 diabetes}

For the purpose of epidemiology, the environment has been described as "all that which is external to the individual human host" [9]. This definition encompasses not only physical and biological agents, but also social, cultural and behavioural factors. It has also been customary to consider innate host characteristics such as biological age under the broad heading of environmental influence, perhaps as a cumulative surrogate for all environmental exposures.

It is beyond the scope of the present article to consider the evidence for individual risk factors for Type 2 diabetes in detail. Suffice it to say that the strongest environmental candidates appear to be the inter-related triad of obesity, reduced physical activity and inappropriate diet [10]. It is not yet clear how these factors cause the disease, if indeed they do, nor whether they do so in the same way in different populations. Evaluation of relative genetic and environmental influences in the aetiology of Type 2 diabetes is further complicated by the likelihood that some of these factors (the tendency to obesity, for example) appear to be subject to a genetic determination which may itself be linked to that of glucose intolerance. Marked obesity is a characteristic of two populations at extremely high risk of Type 2 diabetes (the Pima Indians of Arizona and the Nauruans of Micronesia). However, Jarrett has argued [11] that obesity can account for only a small proportion of diabetes in the United Kingdom, according to relationships established in Israeli data [12]. Whilst risk of diabetes was only modest in Israeli subjects with body mass index of less than 31 , only a very small proportion of the population of the United Kingdom exceed this degree of obesity. Data from the Pimas also indicate little effect of obesity in the absence of family history of the disease [13]. Furthermore, if obesity and glucose intolerance are both manifestations of a disorder characterised by insulin resistance, for which hyperinsulinaemia is the underlying mechanism, obesity may yet prove to be a common effect, rather than a cause, of the metabolic defect.

Age is also an important risk factor for Type 2 diabetes in most populations, but good glucose tolerance is maintained throughout life in some traditional settings. Whilst ageing is an inexorable process, its influence on glucose tolerance is not. Postulating a cumulative effect of known and unknown risk factors on the glucose toler- ance of individuals, it may be that appropriate reduction of other risk factors may lead to a shift in the age-related deterioration in glucose tolerance, to a point where risk of diabetes remains low throughout the natural lifespan, as in the traditional setting. This notion introduces a useful concept: although obvious or widespread risk factors in populations appear the natural candidates for intervention, their reduction may not be practicable (as is the case for age) or widely acceptable (as may be the case for obesity or physical inactivity). In such circumstances, modification of more cryptic component causes may yield better results. This demonstrates the need for a greater understanding of the causal mechanism of diseases such as Type 2 diabetes.

\section{Risk factors from an epidemiological perspective}

To the epidemiologist, a risk factor is an attribute which, when present in an individual, is associated with an increased probability that they will develop a particular disease.

It is important to recognize that risk factors are not necessarily true determinants of the disease. They may be so, but alternatively they may be linked with its occurrence only by virtue of innocent association with the actual components of the causal sequence:

\section{Risk Factor A $\rightarrow$ Disease Risk Factor A

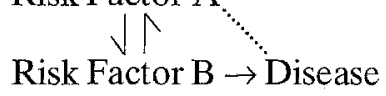

Risk Factor A is causal Risk Factor A is non-causal

For example, an association between coffee drinking and cancer might be observed as a consequence of a tendency for cigarette smokers to drink more coffee than nonsmokers, rather than being a direct effect of drinking coffee. Similarly, the poor outcome of adolescent pregnancies may be shown to be related to parity and socio-economic factors, rather than being a direct effect of biological age. Such confounding is common, and failure to recognize it can lead to mistaken conclusions regarding disease aetiology, and to inappropriate measures for prevention.

Studies in non-Caucasian populations suggest that risk factors for Type 2 diabetes may vary between populations, and between the sexes, within populations [14]: standardised cross-sectional data from field surveys of three populations - Melanesian and Asian Indian residents of Fiji and Micronesians in the Republic of Kiribati - showed an association between the prevalence of diabetes and obesity only in Micronesians and in Indian females. In both Melanesian and Indian males, presence of diabetes was significantly associated only with habitual physical inactivity. In Micronesians of both sexes, an additional association with urban residence was demonstrated, which was independent of the associations with obesity and physical inactivity.

Known risk factors usually explain only a small proportion of any chronic disease in a population, and it seems likely that many as-yet unrecognized influences also have a role in the development of most chronic dis- 

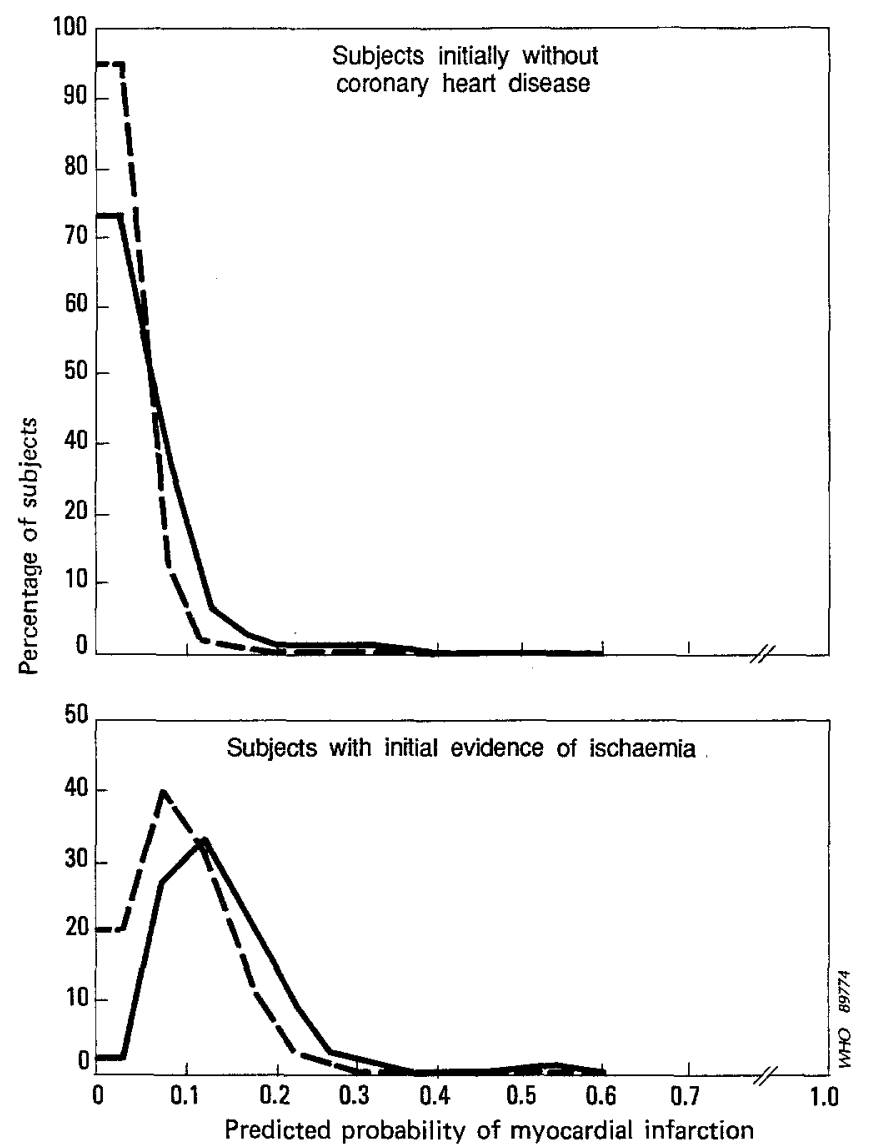

Fig. 1. Distributions of predicted probabilities of developing myocardial infarction in subjects who later developed myocardial infarction (-) and in those who did not (----), according to whether they had initial evidence of ischaemia (Source: R. Heller et al. (1984) Br Med J 288: 1409-1411)

eases, including diabetes. It has been noted in this regard $[15,16]$ that there are large overlaps in predicted probabilities of coronary heart disease in those who do and those who do not later develop the disease (Fig.1). This lack of predictability, based upon the combined multiple logistic scores of several risk factors, is attributed both to the lack of total variation in coronary heart disease morbidity accounted for by the sets of risk factors incorporated in the risk scores, and also to the relative independence of risk factors, which do not tend to cluster at high levels in individuals in the case of heart disease. The small proportion of total variation in heart disease morbidity accounted for by predictive equations implies that there are likely to be other unobserved risk factors which are operating throughout the range of values of the observed risk factors. In addition, if high values for the observed risk factors do not cluster, the risk of disease is likely to be widely spread throughout the population.

These considerations make it clear, once again, that the role of risk factors in disease causation is of fundamental importance when considering disease prevention. Unless the relationship, nature and distribution of all component causes of diabetes were understood, the effect of risk factor reduction could not be predicted reliably in any population.

\section{Alternative strategies for primary prevention}

Epidemiologists studying the development of chronic diseases in populations presently abandoning their traditional way of life have observed that in the case of certain physiological and biochemical characteristics, the adoption of a "modern" lifestyle results in a shift in the whole population distribution of the factor concerned, with a rise in the population mean value [17]. Blood pressure provides a classic example (Fig. 2a). It is this change in the population distribution of risk factors such as blood pressure and blood cholesterol concentration to which the emergence of cardiovascular diseases has been attributed. It has therefore been suggested that appropriate intervention should seek to return the distribution to its baseline characteristics, i.e., to restore biological normality [17]. It is argued that this may be achieved most effectively by appropriate modification of risk factors in the population as a whole, thereby removing the causes of disease incidence. This has been termed the "population approach" to disease prevention.

A comparison of mean values of blood glucose concentration in several Pacific populations at different levels of sociocultural change suggests that this may also apply in the case of glycaemia (Fig. 2b). However, a mean value may be influenced by a relatively small number of observations in the upper tail of the distribution. If the skewing of the distribution due to a small number of high values is the underlying cause of the rise in the population mean value, then a logical action would be to return the distribution to its "biologically normal" (and perhaps also statistically normal) form by eliminating the skewness, rather than by displacing the entire distribution, with or without effect on skewness.

With the exception of the study of bimodality, which has been observed in a few populations at particularly high risk of diabetes, the distributional characteristics of glycaemia have not been given the attention they deserve. This may be because their implications for primary prevention have not been fully appreciated.

A second important prerequisite of the population approach to disease prevention is that the risk of morbid consequences rises throughout a substantial portion of the distribution of the factor concerned, so that the number of events which occur when the factor is only moderately elevated is unacceptable to the community. This relationship is thought to occur in the case of blood pressure and morbid cardiovascular events, for example (Fig.3a). However, in the case of blood glucose concentration, data suggest that the risk of microvascular complications is very low below a value which is diagnostic for diabetes [18]. Such a level is in the upper tail of the population distribution of blood glucose concentration for the majority of the world's communities.

Figure $3 \mathrm{~b}$ shows the prevalence of retinopathy observed in the Pima Indians, superimposed on the distribution of $2 \mathrm{~h}$ plasma glucose concentration in both the Pima Indians, with their very high prevalence of abnormal glucose tolerance, and also that of the general population of the USA. It is clear that for the general population, risk of microvascular complications is small throughout the 

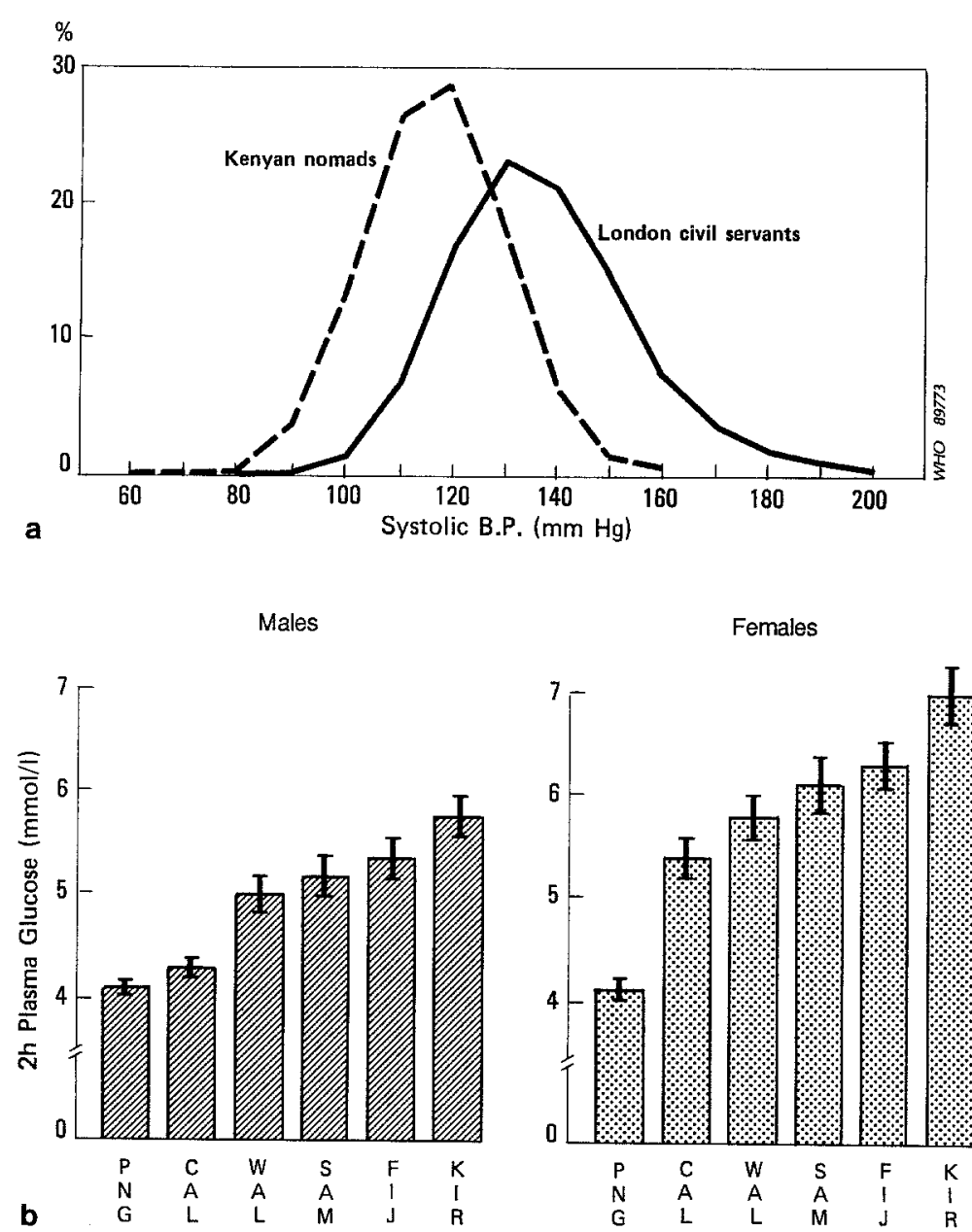

Fig. 2. a Distributions of systolic blood pressure in middle-aged men in two populations (Source: G. Rose (1985) Int J Epidemiol 14: 32-38). b Mean blood glucose concentrations in six Pacific populations, Papua New Guinea, New Caledonia, Wallis Is., W. Samoa, Fiji and Kiribati (Source: H. King et al. (1984) Genet Epidemiol 1: 315-328) population distribution of blood glucose concentration. In these circumstances, the alternative "high risk approach" to prevention may prove more efficient and effective. This strategy aims to identify susceptible subjects, by screening and other case-finding methods, and to intervene selectively to avert the onset of disease.

Type 2 diabetes also confers increased risk of cardiovascular disease, most markedly in females. In addition, cardiovascular disease risk factors are present with greater frequency in diabetic subjects than those with normal glucose tolerance [19]. The independence of cardiovascular risk factors in the general population in both the USA and Finland (the latter suffering a particularly high incidence of coronary events) has been advanced in support of the population approach to the prevention of cardiovascular disease [16]. Invoking the same argument, the higher frequency and high degree of coexistence of cardiovascular disease risk factors in diabetic subjects may indicate merit in an intensified approach to the primary prevention of cardiovascular disease in subpopulations at high risk of diabetes.

It is acknowledged that the population and high-risk strategies are generally complementary [17]. Moreover, there is a moral imperative for both approaches: it is unreasonable to ignore those in society who are especially prone to adverse health outcomes and it is also incumbent upon health authorities to give the public reasoned advice about maximising health for all. Whilst the population approach may be the more appropriate in societies with particularly high genetic susceptibility (in which case the two strategies are effectively the same), the high-risk alternative may have advantages in communities of low/moderate genetic risk, in which the majority of the population will not develop the disease, and in those communities in which diabetes is the predominant noncommunicable disease. The high-risk strategy must also be entertained when there is still uncertainty as to whether the relationship between risk factors and disease is truly causal, as is certainly the case for Type 2 diabetes at the present time.

The high-risk strategy might also prove to have a higher level of acceptability in populations at low/moderate risk. Perception of individual risk has an important influence on public response to advice concerning voluntary behaviours. For example, young Caucasian females do not appear to be heeding anti-smoking advice at present. This may be because their risk of lung cancer is presently low (though likely to rise sharply in future, by cohort effect) and seen as remote in time and therefore of little threat. In contrast, the recent changes in sexual behaviour documented in female prostitutes and male homosexuals show the potential for voluntary change in behaviour when risk of ill-health is perceived as real, and of dire consequence. Whilst it has been argued that subgroups of the population, approached according to a high-risk strategy, 

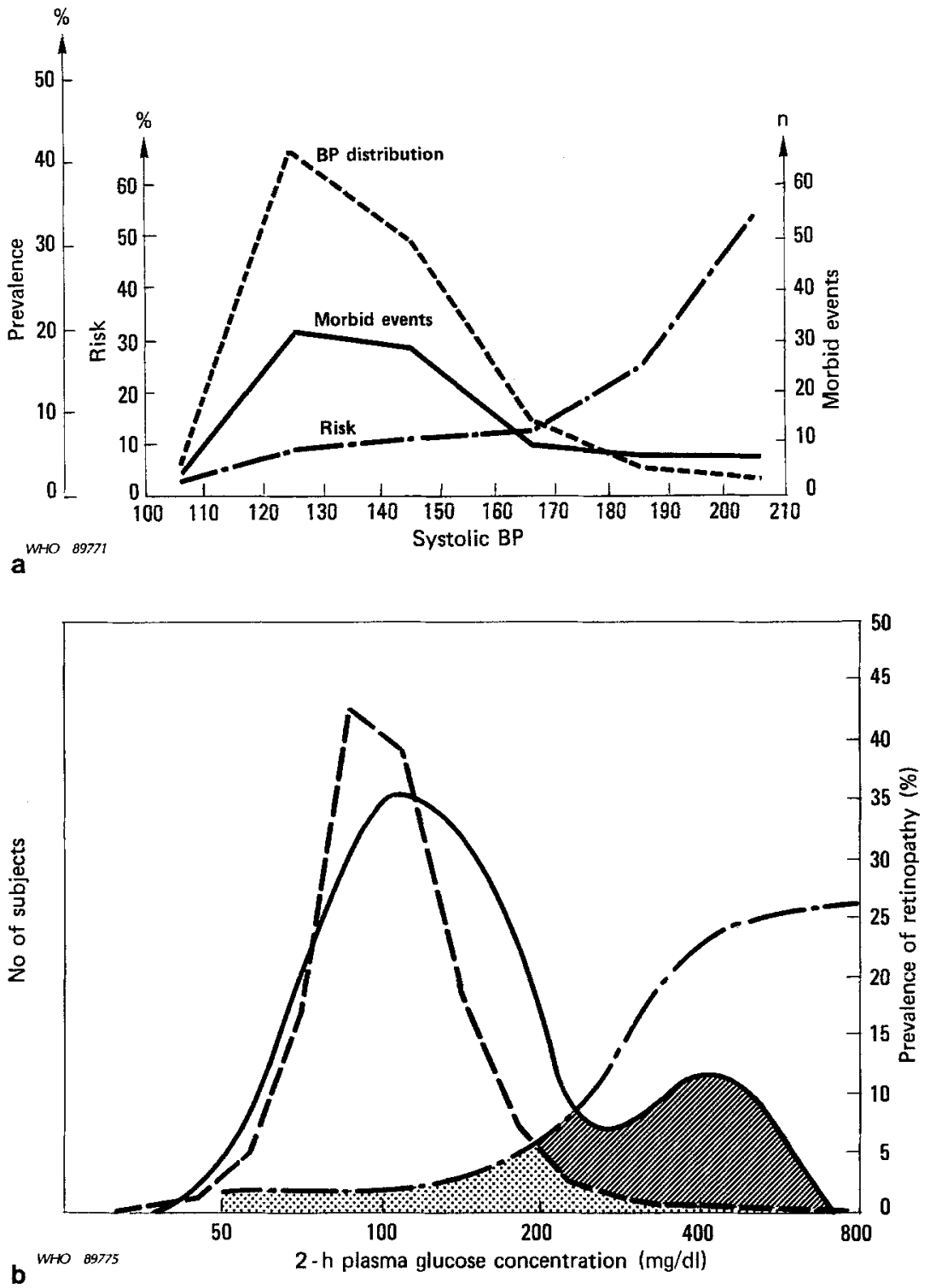

Fig. 3. a Blood pressure distribution, risk of coronary heart disease or stroke, and number of such morbid events in relation to blood pressure during 13.5 years of follow-up of 865 men aged 50 at entry to study (Source: WHO EURO Reports and Studies 98, p 73, 1985. b Blood glucose distribution in the general population of the USA (---) and in the Pima Indians (-) and prevalence of retinopathy in the Pima Indians (-..- - ). (Sources: A. Dorf et al. (1985) Diabetes 25: 554-560 and M. Harris (1989) Diabetes Care 12: 464-474) are less likely to adhere to public health advice than if change is advocated for the whole of society [20], these social changes documented recently in response to the AIDS epidemic suggest that this may not necessarily be the case. Certainly, for obesity, diet and lack of exercise, the most widely accepted risk factors for Type 2 diabetes, nothing short of dedicated motivation is likely to lead to a permanent change in behaviour.

As an example of the high-risk approach to diabetes in practice, screening for impaired glucose tolerance, a category intermediate between the WHO criteria for normal glucose tolerance and diabetes [21] and a marker for future diabetes [22] could be particularly costeffective. Whilst there is continuing controversy surrounding the concept of screening for diabetes in the general population [23, 24], screening for susceptible subjects among the relatives of diabetic patients and other high-risk groups is likely to have a high level of acceptability.

A recent survey of Melanesian children, below the age normally associated with the onset of Type 2 diabetes, demonstrated relative hyperinsulinaemia in an urban as compared with a rural sample, despite similar indices of ponderosity and other cardiovascular risk factors, which might indicate the onset of insulin resistance in youth [25]. Possibly, this may suggest that screening for hyperinsulinaemia early in life might also be a feasible preventive approach in high-risk subgroups within a population.

For most communities, in which individual genetic susceptibility and the frequency and coexistence of risk factors vary, a combination of the population and high risk strategies should be considered. In this way, the health status of the general population, as well as that of subgroups sharing particular characteristics and risk profiles, could be improved simultaneously. In order to determine an optimal distribution of resources, the weight to be attached to each approach would need to be defined, and this would require an in-depth knowledge of the disease processes being considered. Whilst a full consideration of the scientific basis for health policy making is beyond the scope of the present review, the notion emphasises the importance of longitudinal disease surveillance of noncom- 
municable diseases, which enables a prediction of outcome and the calculation of the cost-effectiveness of various mixtures of strategies, including those for primary prevention and curative services.

\section{Conclusion}

There is no doubt that an integrated population approach for the prevention of the major noncommunicable diseases, currently recommended by WHO [1] should be tried and carefully evaluated. Initially, this should be done in selected communities at above-average risk of several of these diseases. Concurrently, a high-risk approach to the prevention of Type 2 diabetes and its complications should also be given serious consideration. Optimal allocation of resources to the two approaches requires a detailed knowledge of disease processes in individual communities and it is clear that for Type 2 diabetes, primary prevention can only be brought about by a substantial and sustained commitment to further aetiological research, with a view to identifying the preventable determinants and the true causal sequence(s).

Acknowledgements. For permission to reproduce the figures the authors are grateful to R. Heller and the British Medical Journal (Fig.1), G.Rose and the International Journal of Epidemiology (Fig. 2a), Genetic Epidemiology (Fig. 2b), World Health Organization (Fig. 3a), A. Dorf and Diabetes, M. Harris and Diabetes Care (Fig. 3 b). Several colleagues offered valuable comment on the manuscript.

\section{References}

1. Epstein FH, Holland WW (1983) Prevention of chronic diseases in the community - one-disease versus multiple-diseases strategies. Int J Epidemiol 12: 135-137

2. Tuomilehto J, Wolf E (1987) Primary prevention of diabetes mellitus. Diabetes Care 10: 238-248

3. World Health Organization (1983) Primary prevention of essential hypertension. Report of a WHO Scientific Group. Technical Report Series 686. WHO, Geneva

4. World Health Organization (1985) Primary prevention of coronary heart disease. Report of a WHO meeting. WHO Regional Office for Europe Reports and Studies 98. WHO Regional Office for Europe, Copenhagen

5. Newman B, Selby JV, King M-C, Slemenda C, Fabsitz R, Friedman GD (1987) Concordance for Type 2 (non-insulin-dependent) diabetes mellitus in male twins. Diabetologia 30: 763-768

6. Neel JV (1962) Diabetes mellitus: a "thrifty" genotype rendered detrimental by progress? Am J Hum Genet 14: 353-362

7. Yamashita TS, Mackay W, Rushforth NB, Bennett PH, Houser $H$ (1984) Pedigree analyses of non-insulin dependent diabetes in the Pima Indians suggest a dominant mode of inheritance. Am J Hum Genet 36: 183S
8. Serjeantson SW, Zimmet P (1984) Diabetes in the Pacific: evidence for a major gene. In: Baba S, Gould M, Zimmet P (eds) Diabetes mellitus: recent knowledge on aetiology, complications and treatment. Academic Press, Sydney, pp 23-30

9. Last JM (1983) A dictionary of epidemiology. Oxford University Press, New York Oxford Toronto, $\mathrm{p} 32$

10. King H (1988) Aetiology. In: Nattrass M, Hale PJ (eds) Non-insulin-dependent diabetes. Baillières Clin Endocrinol Metab 2: 291-305

11. Jarrett RJ (1986) Obesity and Type 2 (non-insulin-dependent) diabetes [letter]. Diabetologia 29: 407-408

12. Modan M, Karasik A, Halkin H, Fuchs Z, Lusky A, Shitrit A, Modan B (1986) Effect of past and concurrent body mass index on prevalence of glucose intolerance and Type 2 (non-insulin-dependent) diabetes and insulin response. Diabetologia 29: 82-89

13. Knowler WC, Pettit DJ, Savage PJ, Bennett PH (1981) Diabetes incidence in Pima Indians: contributions of obesity and parental diabetes. Am J Epidemiol 113: 144-156

14. King H, Zimmet P, Raper LR, Balkau B (1984) Risk factors for diabetes in three Pacific populations. Am J Epidemiol 119: 396-409

15. Heller R, Chinn S, Tunstall Pedoe HD, Rose G (1984) How well can we predict coronary heart disease? Findings of the United Kingdom Heart Disease Prevention Project. Br Med J 288: 1409-1411

16. Kottke TE, Gatewood LC, Wu S-C,Park H-A (1988) Preventing heart disease: is treating the high risk sufficient? J Clin Epidemiol 41: 1083-1093

17. Rose G (1985) Sick individuals and sick populations. Int J Epidemiol 14: 32-38

18. Dorf A, Ballintine EJ, Bennett PH, Miller M (1976) Retinopathy in the Pima Indians. Relationship to glucose level, duration of diabetes, age at diagnosis of diabetes and age at examination in a population with a high prevalence of diabetes mellitus. Diabetes 25: 554-560

19. Wingard DL, Barrett-Connor E, Criqui MH, Suarez L (1983) Clustering of heart disease risk factors in diabetic compared to nondiabetic adults. Am J Epidemiol 117: 19-26

20. Kottke TE, Puska P, Salonen JT, Tuomilehto J, Nissinen A (1985) Projected effects of high-risk versus population-based prevention strategies in coronary heart disease. Am J Epidemiol 121: 697-704

21. World Health Organization (1985) Diabetes mellitus. Report of a WHO Study Group. Technical Report Series 727. World Health Organization, Geneva

22. Saad MF, Knowler WC, Pettit DJ, Nelson RG, Mott DM (1988) The natural history of impaired glucose tolerance in the Pima Indians. N Engl J Med 319: 1500-1506

23. Singer DE, Samet JH, Colet CM, Nathan DM (1988) Screening for diabetes mellitus. Ann Int Med 109: 639-649

24. Davidson MB (1989) Screening for diabetes mellitus [letter]. Ann Int Med 110: 168

25. King H, Alpers M, Finch C, Zimmet P (1989) Future glucose intolerance possibly manifest in youth [Letter]. Lancet II: 1098 1099

Dr. H. King

Division of Noncommunicable Diseases

World Health Organization

$\mathrm{CH}-1211$ Geneva 27

Switzerland 